\section{A relationship between extracapsular involvement and response to steroid treatment in polymyalgia rheumatica: too soon to conclude?}

We read with great interest a recent paper by Mackie et al. ${ }^{1}$ The authors found that an extracapsular pattern of inflammatory involvement on whole-body, multiple-joint MRI was associated with complete glucocorticoid response, higher pretreatment C-reactive protein and serum interleukin-6.

We commend the authors for studying such a challenging disease with advanced imaging and linking it to treatment response. However, we have several concerns. First, it is unclear what criteria were used to distinguish an MRI of 'extracapsular pattern' from that of 'non-extracapsular pattern'. Since at least eight large joints were evaluated bilaterally, are there patients with an extracapsular pattern in certain joints but not in others? How many joints with an extracapsular pattern of inflammation are needed in order to classify a patient as 'extracapsular'? In addition, it is uncertain if the scoring for the degree of inflammation refers to the extracapsular inflammation, intracapsular inflammation or both. Previous studies have shown that bursitis and glenohumeral joint effusions on MRI were seen in almost all patients with polymyalgia rheumatica (PMR) and rheumatoid arthritis (RA) without any difference in frequency between these two groups. ${ }^{2}$ If the degree of extracapsular inflammation was taken into account during the scoring process (as it seems to be the case according to figure 1 of the paper), it is unsurprising that patients with PMR with extracapsular pattern had higher scores than patients with PMR with non-extracapsular pattern and patients with RA. This trend only holds for the large joints because patients with RA had similar or higher scores than patients with PMR in the small joints regardless of extracapsular inflammation.

Second, the outcome measure of patients feeling back to normal' since taking steroids is very subjective. One of the diagnostic criteria for PMR is that patients have to respond to steroids. If a patient diagnosed with PMR does not respond to steroid treatment, then an alternative diagnosis should be considered. $^{34}$ If the patient does not feel 'back to normal', does it mean that the steroid is not having an effect or the patient is unhappy with the side effects of the steroid? The authors stated that the higher percentage of patients reporting 'feeling back to normal' was reflected in better patient-reported function (Health Assessment Questionnaire disability index; HAQ-DI) and fatigue Visual Analogue Scale (VAS) between patients with and without the extracapsular pattern. However, it is important to note that there was a statistically significant difference in the patient-reported pretreatment HAQ-DI and fatigue VAS between the two groups as well. This makes it difficult to evaluate whether or not the post-treatment difference in these two subjective outcomes existed a priori or was a reflection of beneficial effect from treatment.

In summary, the relationship between extracapsular involvement and response to steroid treatment cannot be concluded from such a small cohort. We suggest a clear definition of extracapsular pattern, severity scoring based on extracapsular versus intracapsular inflammation separately and more objective way of assessing patient's response to steroid treatment (eg, reduction of inflammation on MRI).

\section{Li Yang, ${ }^{1}$ Hao Zhou, ${ }^{2}$ Haiyun Tang, ${ }^{3}$ Ashley M Lee, ${ }^{4}$ Harrison X Bai ${ }^{4}$}

${ }^{1}$ Department of Neurology, The Second Xiangya Hospital, Central South University, Changsha, Hunan, P.R. China

${ }^{2}$ Department of Neurology, Xiangya Hospital, Central South University, Changsha, Hunan, P.R. China

${ }^{3}$ Department of Radiology, The First Xiangya Hospital, Central South University, Changsha, Hunan, P.R. China

${ }^{4}$ Department of Radiology, Hospital of the University of Pennsylvania, Philadelphia, Pennsylvania, USA

Correspondence to Dr Li Yang, Department of Neurology, The Second Xiangya Hospital, Central South University, No.139 Middle Renmin Road, Changsha, Hunan 410011, P.R. China; yangli762@gmail.com

Contributors $\mathrm{HXB}$ and $\mathrm{LY}$ drafted the letter; $\mathrm{AML}, \mathrm{HZ}$ and $\mathrm{HT}$ revised the manuscript for important intellectual content; all authors approved the final version.

Competing interests None declared.

Provenance and peer review Not commissioned; internally peer reviewed.

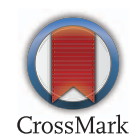

To cite Yang L, Zhou H, Tang H, et al. Ann Rheum Dis 2016;75:e16.

Received 29 November 2015

Accepted 30 November 2015

Published Online First 23 December 2015

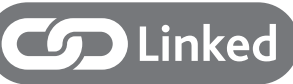

- http://dx.doi.org/10.1136/annrheumdis-2015-208962

Ann Rheum Dis 2016;75:e16. doi:10.1136/annrheumdis-2015-208956

\section{REFERENCES}

1 Mackie SL, Pease CT, Fukuba E, et al. Whole-body MRI of patients with polymyalgia rheumatica identifies a distinct subset with complete patient-reported response to glucocorticoids. Ann Rheum Dis 2015;74:2188-92.

2 McGonagle D, Pease C, Marzo-Ortega $\mathrm{H}$, et al. Comparison of extracapsular changes by magnetic resonance imaging in patients with rheumatoid arthritis and polymyalgia rheumatica. J Rheumatol 2001;28:1837-41.

3 Bird HA, Esselinckx W, Dixon AS, et al. An evaluation of criteria for polymyalgia rheumatica. Ann Rheum Dis 1979;38:434-9.

4 Jones JG, Hazleman BL. Prognosis and management of polymyalgia rheumatica. Ann Rheum Dis 1981;40:1-5. 